\title{
Virtual Nature Environments Based on Fractal Geometry for Optimizing Restorative Effects
}

\author{
Marc van Almkerk \\ University of Twente \\ The Netherlands \\ m.vanalmkerk@student.utwente.nl
}

\author{
Gijs Huisman \\ University of Twente \\ The Netherlands \\ gijs.huisman@utwente.nl
}

\begin{abstract}
Exposure to nature has been shown to have psychological benefits, such as reducing mental fatigue and stress by restoring capacity in directed attention. Moreover, these effects have been found to extend to purposefully designed, virtual representations of nature. Several constructs have been proposed that underlie beneficial characteristics of nature but these constructs have been found difficult to quantify and reproduce consistently. This has made it challenging to create optimized restorative virtual nature environments. Here we present two studies to investigate the role of a more quantifiable parameter - fractals - in the restorative effects of virtual nature. Basic nature scenes featuring trees were generated based on fractal geometry. Results from both an online study including 2D images and a lab study featuring immersive VR showed a small but consistent preference for images with a fractal dimension of 2.3. Results from psychophysiological measurements in the lab study did not show clear effects of the nature scenes on recovery from stress. We discuss the implications of these findings in the light of research towards restoration and give directions for future research.
\end{abstract}

Fractals. Attention Restoration Theory. Virtual Nature. Scenery Assessment. Visual perception.

\section{INTRODUCTION}

Research in environmental psychology suggests that exposure to nature has psychological and emotional benefits to individuals, as it decreases mental fatigue and stress levels [10][23]. Ulrich [30] showed that stressed participants feel significantly better after exposure to images of nature in feelings of affection, friendliness and playfulness, while images of urban environments increased feelings of sadness. Van Rompay and Jol [34] demonstrate that children's creativity is enhanced when they are exposed to nature scenery. Over the past decades, several studies have shown similar, so called, restoration effects; Both in lab and field settings and with various types of surrogate nature such as photos, videos and purposefully designed virtual nature environments (e.g. [24], [31] and [33]).

Findings that promote the use of virtual environments to utilize these effects of restoration [18][33] are particularly interesting, because they imply that virtual nature can be developed that optimizes these health related benefits to viewers. Advances in Virtual Reality (VR) technology open up opportunities for designers to create immersive 3D virtual nature environments which may be especially relevant to improving the well-being of people that do not have direct access to nature, such as office workers, patients in hospitals, astronauts or submariners [10][23]. However, it is an open question [28] which parameters designers of virtual environments should manipulate and in what way to create optimal restorative environments.

In this paper we present two studies to investigate the role of fractal geometry as a potentially relevant parameter that could contribute to the restorative effects of virtual nature environments. In the studies we investigate the effects of both generated 2D images (Study 1) and immersive VR environments (Study 2) in relation to restoration.

\section{BACKGROUND}

In environmental psychology the restorative potential of nature environments is often explained by Attention Restoration Theory (ART) [20][21]. ART theorizes that directing one's attention (i.e. concentrating) requires cognitive effort, of which a person has a limited capacity that can become depleted over time, resulting in mental fatigue. The fatigue can slowly decrease over time, in the absence of directed attention, but the theory suggest that it will be restored faster with exposure to scenery that is 'fascinating' - places that are interesting in themselves, but do not require directed attention to process. Moreover, ART identifies that restorative environments afford a feeling of 'Being Away' from the viewer's everyday life and worries, 
has 'Extent' to constitute a larger whole (i.e. the elements found in the environment are connected to each other) and be 'Compatible' with the viewer's preferences and goals. Overall, it can be argued that environmental preference is made up of two basic needs of information: making sense of the environment and their involvement within the environment [12][20].

To elaborate on this theory, additional studies propose measures to understand the underlying mechanism of these preferences - e.g. studies on specific environments as "water surrounded by vegetation" and "Rock with sparse vegetation" [35] as well as "screening" and "Radiant forest" [12]. Unfortunately, these descriptions are difficult to quantify, making it difficult to reproduce results consistently [28]. Additionally, this makes scenery overall difficult to assess and, specifically for designers, challenging to optimize.

A more quantifiable parameter is proposed by Purcell, Peron and Berto [26], suggesting that a particular type of geometry - fractals - could be involved in the described effects. Forms and shapes in nature, like mountain ranges and coastlines, are highly linked to this type of geometry, while urban environments consists of Euclidean shapes (straight lines) that are rarely found in nature. As the visual complexity of a fractal pattern can be quantified with fractal dimension $D$, this could be a reproducible parameter between scenery. This parameter is a value between 1 and 2, describing how the pattern repeats itself at different magnifications that, combined, will result in the final fractal shape [25]. Several studies investigated the relationship between the visual preference and fractal images [1][14][29] and one study used EEG to measure restoration effects by fractal geometry [13]. All found that the most optimal fractal dimension for these image is a value close to 1.3 or (in some cases) also 1.7 - values that are often found in nature (e.g. waves and clouds have D values of around 1.3, while snowflakes and retinal blood vessels have $D$ values of 1.7) [29].

The exact reason why certain fractal dimensions have visual preference is not fully understood. The main argument is that $D$ values of 1.3 and 1.7 have lots in common with structures in nature, suggesting that the preference for certain fractal dimensions are based on familiarity with these shapes [1]. Another explanation is provided by an eye-tracking study performed by Taylor et al. [29] that made participants view fractal paintings by painter Jackson Pollock as well as computer generated fractals. They found that searching activities with your eyes can be quantified with $D 1.5$, regardless of the fractal dimension that was presented. They hypothesize that the preferences for certain fractal dimensions may come from 'resonance' with our eye trajectories - i.e. certain fractal dimensions could enhance or ease searching activities, leading to a peak in aesthetic appeal.

Within ART, both explanations are combined to articulate why fractal geometry could result in the restoration effects found in nature, within the concept of Perceptual Fluency Account (PFA) [19]. PFA proposes that natural environments are more prone to capture one's attention as they are processed more fluently in comparison to built environments. Although other qualities of nature might play a role in this as well, it is believed that fractals promote this process the most.

Previous fractal-based studies used abstract fractal images or paintings as stimuli and no direct links to ART were made. The purpose of the studies reported here is to explore whether virtual nature environments based on fractal geometry with $D 1.3$ and 1.7 are preferred and whether these environments can elicit restoration effects similar to those found for actual nature. This would help shed light on theoretical questions regarding the beneficial effects of exposure to nature environments. Moreover, fractals could be a parameter for designers to manipulate and optimize virtual nature for human well-being.

\section{OVERVIEW OF STUDIES}

To investigate the potential relation between fractals in nature and ART, two studies were conducted in which participants were exposed to generated virtual nature containing trees with specific fractal dimensions ranging from 2.1 to 2.9. Note, that the fractal dimensions range from 2 to 3 here, as the trees are generated in $3 \mathrm{D}$, in comparison to previous studies that only presented 2D stimuli (i.e. fractal dimensions ranging from 1 to 2 ). In the first study, an online survey was conducted in which participants viewed $2 \mathrm{D}$ representations of virtual nature scenes (i.e. screenshots of the 3D virtual nature scenes) with 5 different fractal dimensions. The aim of this study was to investigate whether virtual nature scenes with fractal dimensions of 2.3 and 2.7 are preferred to scenes with different fractal dimensions, similar to preferences found for 2D stimuli with fractal dimensions of 1.3 and 1.7.In the second study, a lab experiment was conducted in which participants' stress levels were manipulated and physiological data was collected from participants as they viewed two virtual nature scenes with distinctive fractal dimensions (D 2.3 and D 2.9) in VR. Results from the first study were used to select the scenes for the second study.

\subsection{Generation of virtual nature scenes}

3D scenes of different forest environments were made to serve as stimuli in both studies. The focus in the design of each scene was on the canopy of 
the trees as most of the fractals would be present in the branches. This also simplified the set-up, as the focus of the participant would be directed towards the sky rather than an entire landscape. In each scene the parameters of the trees are the same (e.g. location and number of branches), except for their fractal dimension (D) that range from 2.1 to 2.9 in steps of 0.2 (i.e. five different scenes were generated). A script was written in the $3 D$ modeling software Blender $^{1}$ that generated these trees according to the following equation [11]:

$$
D=\frac{\ln b}{\ln \frac{1}{r}}
$$

In which $b$ is the number of branches at each iteration level, while $r$ is the scaling ratio at each iteration level - for the generation of the scenes, b was set at 4 , so that $r$ could be calculated. To make the fractality of the trees somewhat less obvious and more natural, some randomization was added in the angle between branches - a value between 30 and 30 degrees; and to make the trees more visually recognizable they were covered with a bark texture. No leaves were added to the trees to make the scenes focused on the changes in fractal dimension. Each tree was built up from five iteration, so the results were obtained as the tree (not rendered) shown in figure 1.

For study 2, the generated forest scenes could be fully viewed and explored in VR. For study 1, these $3 \mathrm{D}$ scenes were converted to $2 \mathrm{D}$ images as shown in figure 2.

\section{STUDY 1: CROWDSOURCING STUDY OF 2D VIRTUAL NATURE ENVIRONEMENTS}

\subsection{Survey design}

An online survey was conducted to evaluate the generated fractal-based nature with the Perceived Restorativeness Scale (PRS) questionnaire developed by Hartig, Kaiser and Bowler [15] - a questionnaire used to evaluate the restorative effects of a particular nature scene, that is consistent with ART and measures the four factors Being Away, Fascination, 'Coherence' (a subscale of Extent) and Compatibility of a scene. For the survey, only the items belonging to Fascination and Coherence were rated in order to reduce the length of the questionnaire so that the 5 generated nature scenes could be evaluated. In addition to the PRS items, the participants were asked to rate their preference for each scene on a Visual Analogue Scale (VAS). All the five generated scenes (figure 2) were shown to all the participants in random order (within-subject experimental design).

\footnotetext{
${ }^{1}$ https://www.blender.org/
}
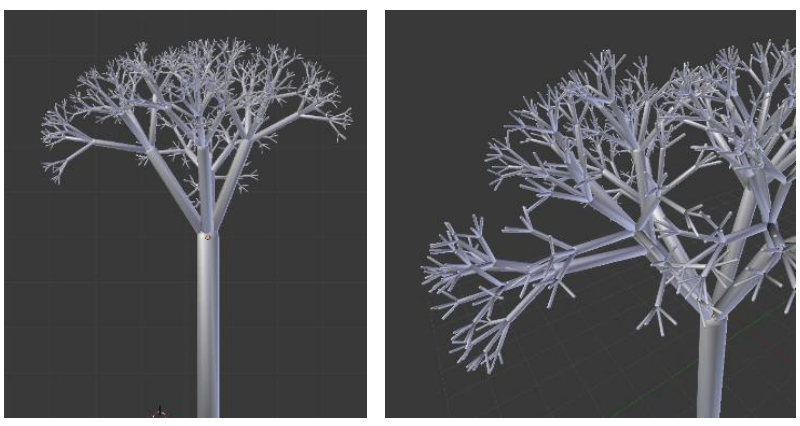

Figure 1: A generated tree ( $D=2.3, b=4$, iteration $=5)$ in blender, without bark texture.

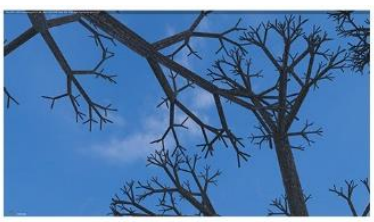

$\mathrm{D}=2.1$

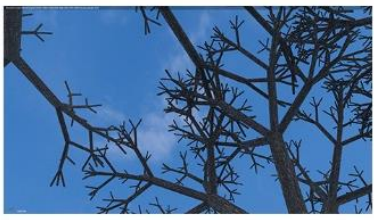

$D=2.5$

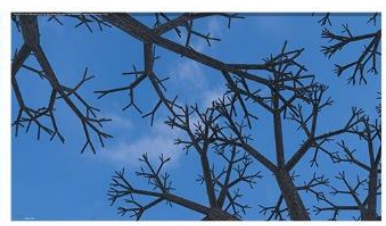

$\mathrm{D}=2.3$

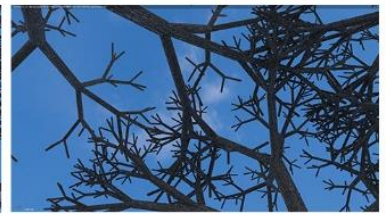

$\mathrm{D}=2.7$



Figure 2: The five generated virtual nature scenes based on fractal dimensions used for study 1 ( $D=2.1$ till $D=2.9$ in steps of 0.2).

\subsection{Procedure}

For each scene participants rated the 12 items taken from the PRS questionnaire on a 7-point Likert scale to indicate the extent to which the given statement described their experience of the scene ( $0=$ Not at all; $6=$ Completely $)$. To indicate their preference for each scene, the VAS was presented through a slider bar that went from -100 to 100 in steps of 1 and was by default on 0 . Furthermore, two control statements were randomly distributed within the PRS questionnaire items as a check for the accuracy of participants' responses [3]. In these statements the participants were asked to select a specific rating on the scale.

\subsection{Participants}

The survey was distributed through the crowdsourcing website Crowdflower ${ }^{2}$ to acquire only native English speaking participants. In total 237 participants completed the questionnaire but not all were included in the results as participants did (i) not correctly answered the control questions 
or (ii) their response time was too quick (the mean of the overall response time minus the standard deviation) or (iii) their response time was too long (the mean of the overall response time plus three times the standard deviation). As the questionnaire was relatively long, longer response times were permitted with greater leniency (e.g. longer response times can be explained by participants being briefly interrupted while completing the questionnaire). Moreover, some participants were removed manually if more than half of the ratings had the same value of 0 or 6 and were relatively close to the response time limits and/or the VAS had low variance between the different scenes (e.g. only -100 or 100 ). These response patterns are a general indication of 'Smart Deceivers' [3]. After this filtering procedure, 119 participant were included in the analyses. The majority of the participants included had an age between 30 to 49 $(n=67)$, with an equal distribution of younger and older participants ( $\mathrm{n}=26$ and 27 , respectively). Moreover, the majority of participants was female $(\mathrm{n}=68)$.

\subsection{Results}

Table 1 shows the results of a reliability analysis on the PRS questionnaire. The results show that for each scene there is a high internal consistency between the items; for both subscales Fascination and Coherence. For subscale Fascination this is consistent with previous studies, while Coherence has a higher internal consistency (greater than .8) than typically found in other research [18][22].

Table 1: Cronbach's Alphas for each scene for both subscales Fascination and Coherence.

\begin{tabular}{|l|c|c|}
\hline Scene & $\begin{array}{c}\alpha \text { Fascination } \\
(8 \text { items })\end{array}$ & $\begin{array}{c}\alpha \text { Coherence } \\
(4 \text { items })\end{array}$ \\
\hline $\mathbf{D = 2 . 1}$ & .934 & .898 \\
\hline $\mathbf{D = 2 . 3}$ & .941 & .870 \\
\hline$D=\mathbf{2 . 5}$ & .925 & .894 \\
\hline$D=2.7$ & .925 & .920 \\
\hline$D=2.9$ & .907 & .916 \\
\hline
\end{tabular}

The subscale scores for Fascination and Coherence were calculated for each participant (for every scene) by taking the mean of the items belonging to the respective subscale. Subsequently, the means were averaged over all the participants to calculate subscale scores of the particular scenes, resulting in Figure 3 for Fascination and Figure 4 for Coherence.

Figure 3 shows that the scene with fractal dimension $D=2.3$ contains the highest Fascination $(M=3.03$, $S D=1.34)$, and $D=2.9$ scores the lowest $(M=2.72$,

\footnotetext{
${ }^{2}$ https://www.crowdflower.com/ (rebranded in March 2018 to 'Figure Eight')
}

$\mathrm{SD}=1.18)$ with a slight increase at fractal dimension $\mathrm{D}=2.7(\mathrm{M}=2.85, \mathrm{SD}=1.27)$. $\mathrm{D}=2.1$ and $\mathrm{D}=2.5$ have a slightly higher score then $\mathrm{D}=2.9(\mathrm{M}=2.79, \mathrm{SD}=$ 1.27 and $M=2.75, S D=1.19$ respectively). However, a repeated measures ANOVA revealed no statistically significant differences for Fascination $(F(3.11,367)=2.58, p=.051, \eta p 2=.021)$.

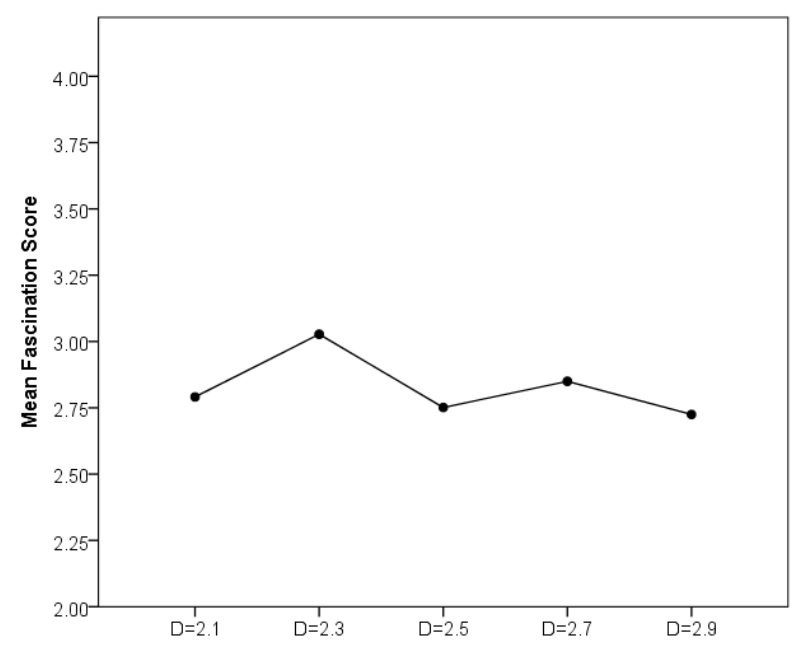

Figure 3: Mean ratings for PRS subscale Fascination for each virtual nature scene.

Figure 4 shows that the Coherence is going down if the fractal dimension is increased - with fractal dimension $\mathrm{D}=2.1(\mathrm{M}=4.57, \mathrm{SD}=1.25)$ and $\mathrm{D}=2.3$ $(\mathrm{M}=4.56, \mathrm{SD}=1.09)$ having the highest Coherence score, and $D=2.9(M=2.90, S D=1.68)$ the lowest. A repeated measures ANOVA revealed a statistically significant difference for the Coherence scores $(F(3.17,374)=71.4, p<.001, \eta p 2=.575)$. A post hoc test with Bonferroni correction revealed a significant difference between every scene, except between $D=2.1$ and $D=2.3(p=1)$.

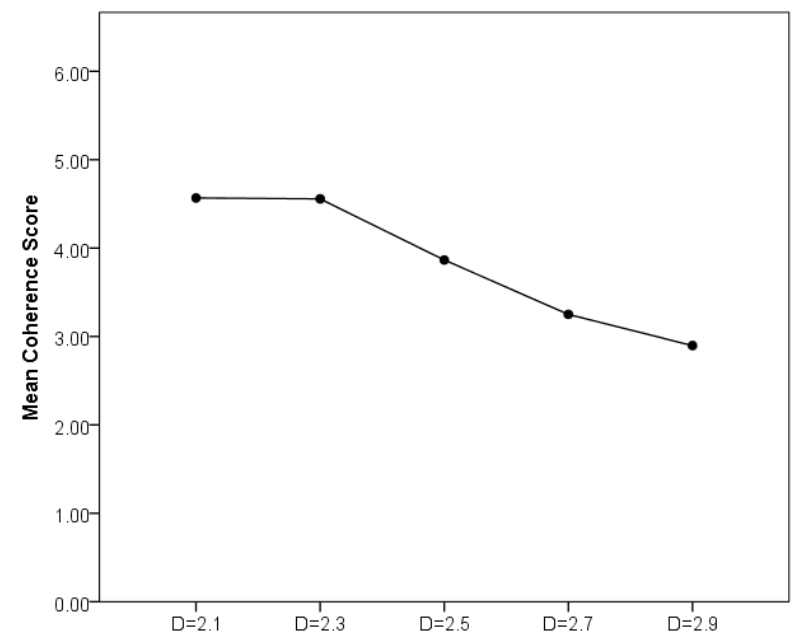

Figure 4: Mean ratings for PRS subscale Coherence for each virtual nature scene. 
Lastly, Figure 5 shows the means of the preference ratings for each scene. Overall, participants preferred lower fractal dimensions over high fractal dimensions. $\mathrm{D}=2.1$ shows to be a relative neutral scene $(M=1.93, S D=42.7)$ while $D=2.9$ is preferred the least $(M=-16.62, S D=49.7)$, but with a clear preference peak at $\mathrm{D}=2.3(\mathrm{M}=7.44$, $\mathrm{SD}=48.8$. . A repeated measures ANOVA revealed a statistically significant difference for preference $(\mathrm{F}(3.08,364)=12.4, \mathrm{p}<.001, \mathrm{np} 2=.095)$. A post hoc test with Bonferroni correction revealed a statistically significant difference between the scene with $D=2.3$ and scenes with a higher fractal dimension $(p=.016, p<.001$ and $p<.001$, respectively).

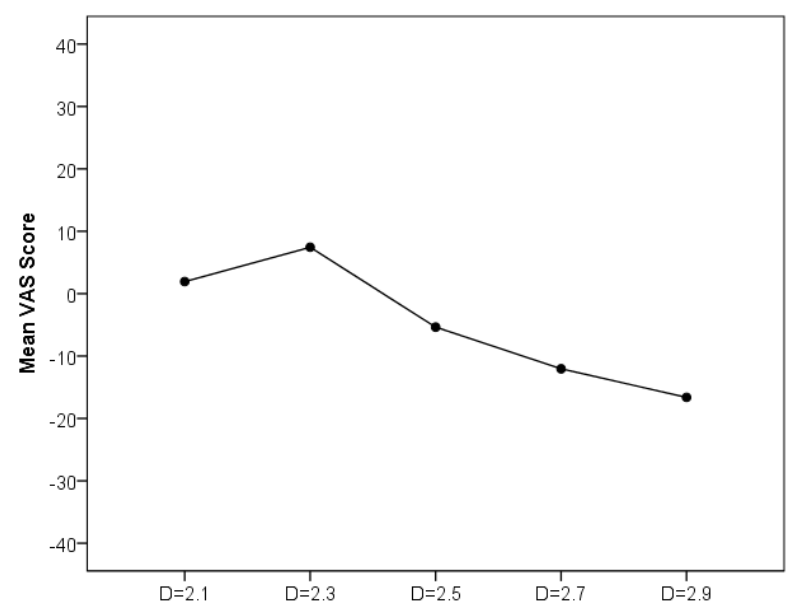

Figure 5: Mean preference ratings for each virtual nature scene.

\subsection{Preliminary conclusion}

The results of the survey show that $D=2.1$ and $\mathrm{D}=2.3$ are preferred over the other, higher, fractal dimensions. An indication for the preference of $\mathrm{D}=2.3$ is also visible in the PRS subscale Fascination, although no statistically significant differences were found. The ratings for the subscale Coherence show a different pattern as they go down when the fractal dimension increases. This is, however, consistent with the behavior of fractals as more overlapping happens when $D$ increases, causing more 'visual chaos'. Nonetheless, fractal dimension $\mathrm{D}=2.3$ is still considered quite coherent (on the same level as $D=2.1$ ) and ratings only go down for higher fractal dimensions.

In contrast to previous studies [29], no clear preference was found for $D=2.7$; although higher ratings for Fascination were found for the scene with this specific fractal dimension. Instead, the results show that the participants gave higher preference ratings for $D=2.1$. The reason for this can be that the scene with $D=2.1$ is not distinctive enough from $D=2.3$, as the $3 D$ forest was converted to a $2 \mathrm{D}$ image potentially making the fractal pattern less visible to the viewer.
Overall the results of the survey are consistent with previous research on fractal dimensions in 2D stimuli [1][13][14][29] and suggest that a fractal dimension of 2.3 in 3D geometry is, as well, a special case that could potentially elicit the most restoration. In contrast, $\mathrm{D}=2.9$ has the lowest score in all the metrics, suggesting that this scene has the lowest restorative potential. Hence, for Study 2, these two scenes were used as the experimental stimuli to examine the virtual nature in relation to stress.

\section{STUDY 2: REDUCING STRESS IN IMMERSIVE VIRTUAL NATURE ENVIRONMENTS}

\subsection{Study design}

A lab study was conducted in which participants were immersed in a VR environment. Participants underwent a stress induction test to increase their psychological stress levels and physiological arousal. The procedure of the Sing-a-Song Stress Test (SSST) was chosen as a relative easy but effective method to induce stress [6]. After the stress was induced, participants were shown both a control and nature scene (random order per participant) in which the control scene served as a baseline for stress recovery without stimuli. Between participants the nature environment could either be a nature scene with high or low restorative potential in order to determine if fractal dimensionality would influence recovery from stress. As the results from study 1 suggested that $\mathrm{D}=2.3$ has the highest restorative potential and $\mathrm{D}=2.9$ the lowest, these two scenes were used as the between subjects conditions. For the control scene, participants were only shown the blue sky box that is present behind the branches of the tree (like the background used in the various scenes of figure 2).

\subsection{Measurements}

During the entire experiment, the Electrodermal Activity (EDA) and Electrocardiography (ECG) of the participants were recorded, to measure physiological indicators of stress [8][32]. The EDA recordings where used to count Skin Conductance Responses (SCRs) of the participants, reflecting the activity in the sympathetic nervous system (SNS) that is responsible for the body's fight-orflight response. More activity in the SNS indicates higher stress related physiological arousal. The ECG recordings were used to calculate the Heart Rate Variability (HRV) - the amount the heart varies in-between heart beats. A high HRV is associated with good health, while a low HRV is associated with illness or stress. Moreover, next to the physiological measurements, participants were asked to complete the PRS questionnaire, now including all the 26 items. 


\subsection{Procedure}

Figure 6 shows the procedure followed for both conditions. Participants were invited to an office room and were only told that they would do an experiment involving VR but were not informed about its context or the stress test. It was made clear to participants that they could withdraw from the study at any time if they felt uncomfortable. Participants were asked to sign a consent form describing the procedure but, again, not the purpose of the experiment. Next, the EDA and ECG equipment (Biosemi Mark II) was attached and participants were asked to lie down on the floor facing the ceiling. This would result in a viewpoint in VR of the canopy of the trees as if one was lying down under beneath them. The view was similar to that in study 1. Subsequently, the signal from the EDA and ECG equipment was checked to make sure the data was recorded correctly. Participants were clearly instructed not to talk and move for the duration of the study. Finally, the participant would be equipped with a HTC Vive headset to immerse the person in VR.

The study started with a baseline measurement of 120 seconds, followed by four mental (thinking) tasks in a black surrounding, visible through the VR headset, so participants were not distracted by other visual stimuli. The participants were asked to relax and follow the tasks that appeared in front of them in the virtual space. Tasks included "thinking about animals that start with the letter $\mathrm{P}$ " and "objects that one can find in the kitchen". Each task took 30 seconds (in which the text appeared for 10 seconds) and a timer in the corner of their view showed how much time was left.

After the baseline measurement, the main SSST task was given to induce stress: participants had 20 second to think about a song that they could sing out loud for 30 seconds after the timer hit zero. As soon as the timer hit zero, the participants were instructed to sing the song they could come up with [6]. Subsequently, after they were done singing, the participants would be informed to relax again (and remembered not to move); and were placed either in the control scene or conditional nature scene (random order) for 40 seconds to measure the physiological response to the stimuli. During this period no timer was present so the participants could only focus on the canopy of the fractal trees in the virtual scene. Once the 40 seconds were over, the participants were placed in second scene (either the control seen or nature scene, depending on what they saw first) for another 40 seconds. Between the exposure of the two scenes there was a 14 seconds rest.

Once the two scenes were shown, the EDA and ECG equipment was removed. At this point participants were still wearing the VR headset and were placed in the nature scene matching their experiment condition for a second time. Participants were asked to explore the scene in a standing position and get an impression of the scene for two minutes. This was done to ensure that participants that got the fractal scene directly after the stress induction, answered the PRS questionnaire with that scene in mind and not the control scene that was shown second. Finally, the VR headset was removed and the participant was asked to complete the PRS questionnaire.

\subsection{Participants}

The total number of measured participants was 40 , 20 for condition $\mathrm{D}=2.3(\mathrm{M}=23.2, \mathrm{SD}=1.85)$ as well as $\mathrm{D}=2.9(\mathrm{M}=23.4, \mathrm{SD}=2.68)$. However due to faults in the measurements only 36 participants were included in the analyses $(n=19$ for $D=2.3$ and $n=17$ for $D=2.9$ ). Faults occurred because electrodes were not properly placed, showing artifacts in the signals. All participants were recruited through convenience sampling and were students from a Dutch university but included many non-Dutch students $(n=29)$ like Italian, Indian and Chinese. 6 of the 20 participants that were exposed to the $D=2.3$ scene were female; while for the $D=2.9$ scene this number was 4 .

\subsection{Data Preprocessing}

\subsubsection{SCR}

The EDA data was recorded at $2048 \mathrm{hz}$ but was down sampled to $10 \mathrm{hz}$ to remove noise and small measurement artifacts. A min-max analysis (trough-to-peak) was computed over the entire measurement to identify SCRs. subsequently, the number of SCRs were calculated during the presence of the stimulus to quantify the activity of the SNS [5].

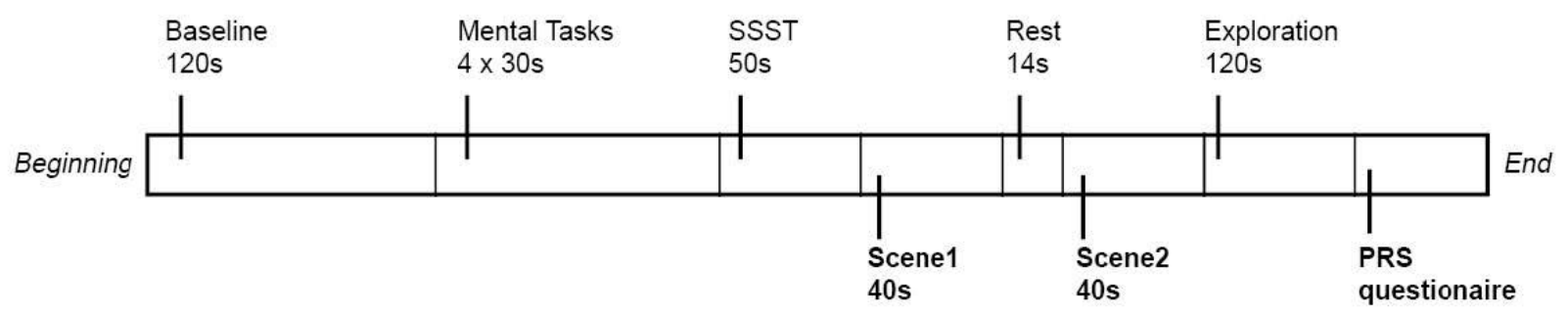

Figure 6: Timeline of the procedure. EDA and ECG were recorded at the beginning of the experiment till after the second scene, while PRS questionnaire was conducted after the physiological measurements were done. 
Using the outlier labeling rule [17] with a 2.2 interquartile range (IQR) for determining the outer fences [16], two possible outliers where identified. Both cases belonged to the $\mathrm{D}=2.3$ condition, having a high SCR count for the nature stimuli. After further inspection, both cases were discarded from the data as the noise in the signal had an excessive influence on the trough-to-peak analysis.

\subsubsection{HRV}

From the recorded ECG signal the low-frequency component was removed and the measurements were analyzed for identifying the $R-R$ peaks. This was done by finding the maxima in a window filter over the entire exposure period of the stimuli. Using the R-R peak intervals, the HRV was calculated with the Root Mean Square of Successive Differences (RMSSD) time-domain method [7] and was computed for each participant for each given stimulus. This method was chosen as Salahuddin et al. [27] showed that HRV can be calculate reasonably accurately using this method in short time periods - i.e. within 40 seconds, instead of the usual 5 minutes.

The outlier labeling rule (with a 2.2 IQR) was computed on the HRV data as well to determine possible outliers. Three cases were identified as outliers, of which two belonged to the $D=2.9$ condition and one to $D=2.3$. After inspection of the raw data, these measurements were excluded from the statistical analysis as they seemed compromised by artifacts.

\subsection{Results}

\subsubsection{Statistical analysis of stress induction}

In order to confirm that the stress induction was effective, EDA and HRV were analyzed during the period of administering the SSST. To confirm EDA levels, the measurements were converted to Zscores [4] and the slope of the signal was analyzed. Identifying SCRs was unnecessary for this scope, as EDA levels should increase in general when these responses happen. For each participant the moment right before the SSST started was taken $(M=-1.04$, $\mathrm{SD}=.353$ ) as well as right before the first stimuli was presented $(\mathrm{M}=1.32, \mathrm{SD}=.598)$. The data show that the EDA levels increased for every participant. Using a repeated measure ANOVA it was found that the EDA increased significantly from the baseline $(F(1,36)=596, p<.001, n p 2=.943)$. To confirm HRV, a similar method was used; however instead of using only one point, a time span of 40 second was taken before the stress induction $(\mathrm{M}=37.1 \mathrm{~ms}, \mathrm{SD}=26.1)$ and 40 seconds to the end of stressor $(M=26.6 \mathrm{~ms}, \mathrm{SD}=11.1)$. The data show that for 20 of the 35 participants the HRV decreased and by means of a repeated measure ANOVA, it was found that the decrease in HRV was statistically significant $(F(1,34)=596, p=.023, n p 2=.144)$. These findings correspond with earlier studies and are indicative of a successful induction of stress [9][33].

\subsubsection{Statistical analysis of psychological measurements}

Figure 7 shows the mean SCR for the control and nature stimuli in each condition. Both the control (M $=5.13, \mathrm{SD}=6.51)$ and nature stimuli $(\mathrm{M}=4.31, \mathrm{SD}$ $=2.89)$ in the $D=2.3$ condition are lower than the $\mathrm{D}=2.9$ condition $(\mathrm{M}=7.12, \mathrm{SD}=5.30$ and $\mathrm{M}=$ $4.59, \mathrm{SD}=3.57$, respectively). This indicates that, overall, participants in the $D=2.3$ condition felt less stressed. Additionally, in both conditions the SNS seemed less active in presence of the virtual nature compared to the control stimuli, due to a lower mean of SCR. Nonetheless, the data show high standard deviations, suggesting that individual differences play an important role in how the stimuli are perceived.

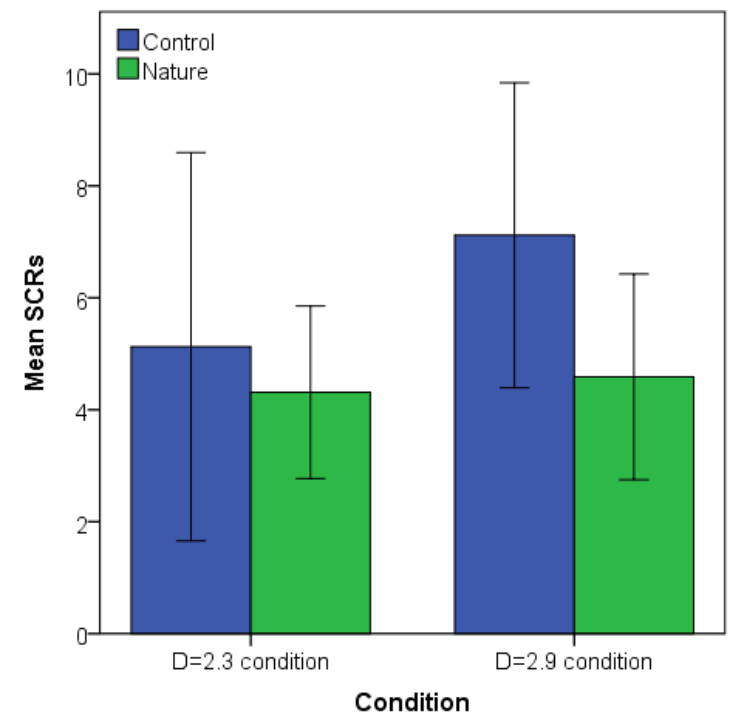

Figure 7: Mean SCR for the control and nature stimuli in both conditions.

A mixed ANOVA (2x2) with the fractal conditions as independent factor and the SCR as within-subject factor was used to analyze the results statistically. The analyses reveal no statistically significant differences for the SCR $(F(1,31)=3.29, p=.080$, $\eta \mathrm{p} 2=.096)$, fractal dimensions $(\mathrm{F}(1,31)=.670, \mathrm{p}=$ $.419, \mathrm{np} 2=.021)$ or the interaction between SCR and condition $(F(1,31)=.867, p=.359, n p 2=.027)$.

Figure 8 shows the mean HRV for both conditions. Overall, the same conclusion could be draw as with the SCR analysis. The HRV in the control $(\mathrm{M}=$ $44.1 \mathrm{~ms}, \mathrm{SD}=22.2)$ and nature stimuli $(\mathrm{M}=$ $45.7 \mathrm{~ms}, S D=21.6$ ) for the $D=1.3$ condition are both higher relative to the $D=1.9$ condition $(M=$ $37.0 \mathrm{~ms}, \mathrm{SD}=17.4$ and $\mathrm{M}=38.1 \mathrm{~ms}, \mathrm{SD}=18.9$, respectively); and people seemed less stressed in the virtual nature scenes compared to the control scenes. Hence, the HRV also suggests that during the exposure of $D=1.3$ fractals, participants felt less 
stressful. Moreover, the HRV seemed to be influenced by individual differences as well, due to the relative large standard deviations.

A mixed ANOVA ( $2 \times 2)$ with the fractal conditions as independent factor and the HRV as within-subject factor was used to analyze the results. The analyses revealed no statistically significant differences for $\operatorname{HRV}(F(1,30)=.339, p=.564, n p 2$ $=.011)$, fractal dimension $(F(1,30)=1.18, p=.287$, np2 $=.038)$, or the interaction between HRV and condition $(F(1,30)=.009, p=.923, n p 2=.000)$.

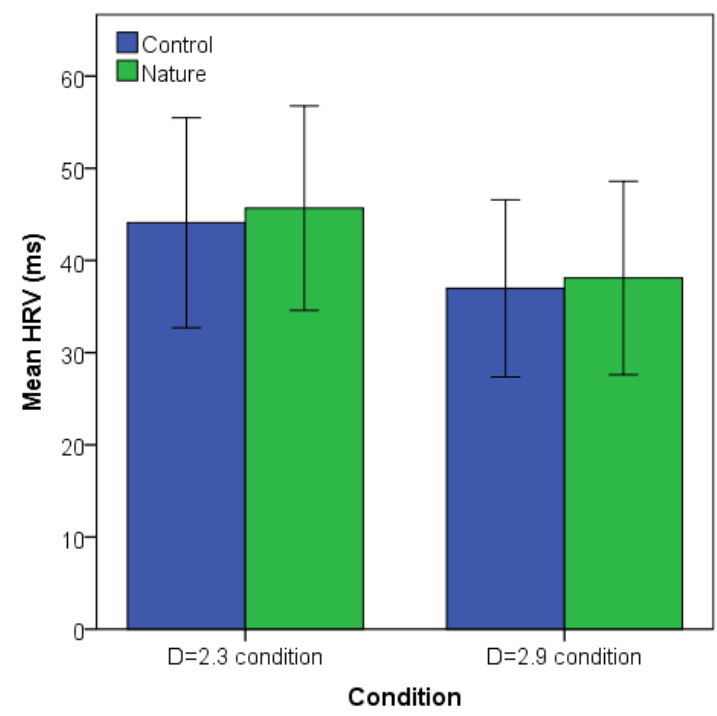

Figure 8: Mean HRV for the control and nature stimuli in both conditions.

\subsubsection{Analysis of PRS questionnaire}

Table 2 contains the results of a reliability analysis for the PRS questionnaire, showing that Fascination as well as Being Away had a high internal consistency. However, contrary to the results of study 1, Coherence has a very low internal consistency within this study. In addition, Compatibility also shows to have a relatively low internal consistency. Removing certain items from the respective subscales did not increase the alpha.

Table 2: Cronbach's Alphas for each PRS subscale in both conditions.

\begin{tabular}{|c|c|c|}
\hline Subscale & $\begin{array}{c}\mathrm{D}=2.3 \\
\text { Nature }\end{array}$ & $\begin{array}{c}\mathrm{D}=2.9 \\
\text { Nature }\end{array}$ \\
\hline Being Away & .794 & .896 \\
\hline Fascination & .903 & .876 \\
\hline Coherence & .222 & .482 \\
\hline Compatibility & .626 & .567 \\
\hline
\end{tabular}

Figure 9 shows the means of the four subscales Being Away $\left(\mathrm{M}_{\mathrm{D}=2.3}=3.75, \mathrm{SD}_{\mathrm{D}=2.3}=1.14 ; \mathrm{M}_{\mathrm{D}=2.9}=\right.$ 3.22, $\left.\mathrm{SD}_{\mathrm{D}=2.9}=1.28\right)$, Fascination $\left(\mathrm{M}_{\mathrm{D}=2.3}=3.37\right.$, $\left.\mathrm{SD}_{\mathrm{D}=2.3}=1.32 ; \mathrm{M}_{\mathrm{D}=2.9}=3.13, \mathrm{SD}_{\mathrm{D}=2.9}=1.24\right)$ ), Coherence $\left(\mathrm{M}_{\mathrm{D}=2.3}=5.15, \mathrm{SD}_{\mathrm{D}=2.3}=.646 ; \mathrm{M}_{\mathrm{D}=2.9}=\right.$
4.83, $\left.\left.\mathrm{SD}_{\mathrm{D}=2.9}=.816\right)\right)$ and Compatibility $\left(\mathrm{M}_{\mathrm{D}=2.3}=\right.$ $\left.3.49, \mathrm{SD}_{\mathrm{D}=2.3}=.875 ; \mathrm{M}_{\mathrm{D}=2.9}=3.18, \mathrm{SD}_{\mathrm{D}=2.9}=.738\right)$ ) independently, as well as the overall PRS mean $\left(\mathrm{M}_{\mathrm{D}=2.3}=3.94, \mathrm{SD}_{\mathrm{D}=2.3}=.754 ; \mathrm{M}_{\mathrm{D}=2.9}=3.59, \mathrm{SD}_{\mathrm{D}=2.9}\right.$ $=.741$ ) by taking the average over the four subscales [26]. A difference is visible for all the ratings between the two conditions, all favoring fractal dimension $D=2.3$, suggesting that this condition has more restorative potential. However, due to the low internal consistencies for Coherence and Compatibility, the means for the overall PRS score and both subscale should be interpreted with care. Nonetheless, the results, as obtained within this study, do show consistencies with the result of study 1 as well as the findings of other studies [1][13].

An independent samples t-test was performed for the restorative scale, but revealed no significant different $(\mathrm{t}(38)=1.49, \mathrm{p}=.144$ (two-tailed), $\mathrm{d}=$ .047). Individual independent t-tests with a significance assessed at alpha/4 for each subscale, also revealed no significant difference for Being Away (t $(38)=1.38, \mathrm{p}=.174$ (two-tailed), $\mathrm{d}=.043$ ), Fascination ( $\mathrm{t}(38)=.586, \mathrm{p}=.561$ (two-tailed), $\mathrm{d}=$ .019 ), Coherence (t $(38)=1.40, p=.170$ (twotailed), $\mathrm{d}=.044$ ) and Compatibility (t $(38)=1.24, \mathrm{p}$ $=.224$ (two-tailed), $\mathrm{d}=.039$ ). The same analysis was also performed on all the items corresponding to Coherence and Compatibility, but these results revealed no significance differences as well (significance assessed at alpha/4 for Coherence and alpha/9 for Compatibility).

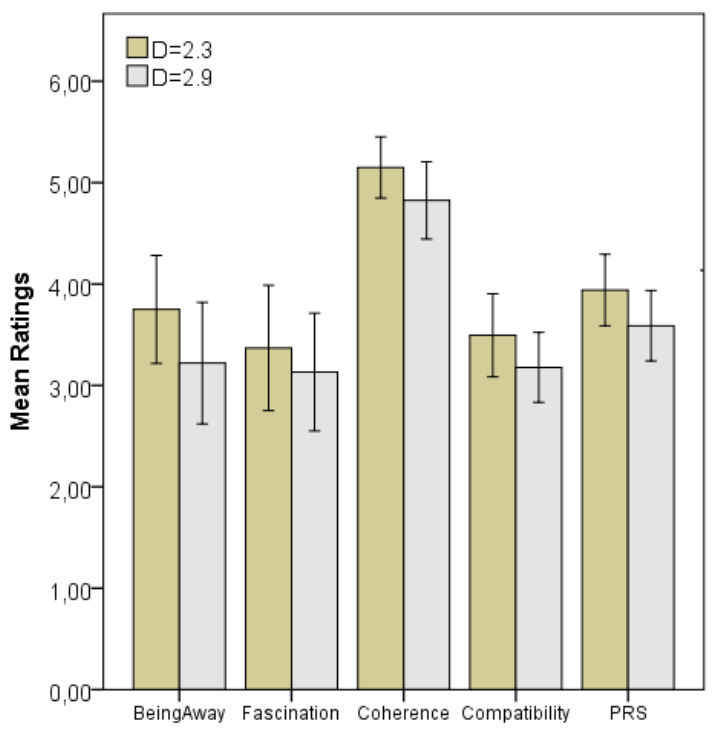

Figure 9: Mean ratings for each subscale of the PRS questionnaire, as well as the overall mean PRS score for both conditions.

\section{CONCLUSIONS AND DISCUSSION}

Results from both studies presented here provide some tentative evidence for the involvement of fractal dimensions in restorative effects of virtual 
nature environments. In study 1, a preference was found for the scene with a fractal dimension of $\mathrm{D}=2.3$. This finding was supported by the Coherence sub-scale of the PRS in that 2.3 (as well as 2.1) were rated as more coherent than scenes containing other fractal dimensions. Both these findings are supported by literature on the restorative effects of surrogate and actual nature scenery [18][23][24] [31][33] and shows that a visual preference for 2.3 is present in 3D geometry. In addition, the data from study 2 show a trend towards stronger recovery from stress after exposure to virtual nature with a fractal dimension of 2.3 compared to a fractal dimension of 2.9, as indicated by physiological measures (on average lower EDA and higher HRV for the scene with $D=2.3$ ) and PRS ratings (overall higher mean ratings for the scene with $D=2.3$ ). However, the differences between the virtual nature scenes with different fractal dimensions in study 2 were not statistically significant. Both studies reported here thus do not provide strong evidence for the role of fractals in restorative effects of virtual nature scenes from an ART perspective. Two aspects related to the design of the current studies and stimuli can provide some explanation for the lack of robust effects.

First, it is not unthinkable that the overall effect size of fractals dimensions presented in relative isolation, that is, implemented in basic 3D models of trees, on restoration is small. If there would indeed be a small true effect for fractals, the sample size of study 2 was too small to detect it. Nevertheless, the sample size of study 1 was substantial, yet only a statistical difference was found for Coherence, but not for Fascination. An explanation is that the PRS scale, which is specific to the ART perspective on restorativeness of nature scenery, is not well suited to measure impressions of fractal-based nature scenes. Some evidence for this is found in the fact that in study 1 statistically significant differences between the scenes were found for the general preference ratings.

Second, the minimalistic design of the virtual nature might have made it difficult for participants to judge the scenes in accordance with ART. For study 1, this appeared to have been less of an issue as participants were only presented with one viewpoint of the canopy. However, the minimalistic design of the scenes might have been made more apparent by the fact that in study 2 participants were allowed to looking around the scene before completing the PRS scale. Participants might have based their judgements on the overall appeal of the view, rather than focus on the fractals presented in the canopy of the trees. Since, apart from the trees, the landscape was relatively barren, this might have influenced PRS ratings and could explain the low internal consistency for Coherence in study 2. Furthermore, the design of the trees themselves could be improved upon in future studies. From a qualitative point of view the trees could be seen as "dead trees" (as mentioned by a few participants) that do not necessarily evoke Fascination in the viewer. Evidence for this can be found in the relatively low mean scores for Being Away and Fascination in study 2 , as nature scenes normally tend to score higher on those scales [26].

These limitations should, however, be viewed in relation to previous research on fractals and ART. The here presented studies offer a starting point, as 3D fractal shapes have, to the best of the authors' knowledge, not yet been studied from an ART perspective. Nature environments and fractal geometry are generally visually complex, and are therefore often studied holistically using relatively general measures (e.g. preference) [1][14][29]. Factors contributing to the build-up of nature environments can be isolated to study their individual effects, as exemplified by studies on abstract fractal visualizations. However, in order to make statements about which measurable factors contribute to restorative effects of nature and in order to enable the design of optimized nature scenery, the two lines of research need to be brought together. The here presented studies are an attempt in this direction. The studies show that it is possible to obtain relatively robust preference ratings for virtual nature environments designed with fractals (study 1). However, the results from study 2 illustrate that such preference ratings might not translate straightforwardly to actual effects of virtual nature environments on restoration.

Based on the findings from the here presented studies, it is likely that fractal dimensions contribute to restorative effects of nature to some extent, but that they do so in combination with other aspects of nature. If we consider fractal patterns in more depth, Aks and Sprott [1], for example, identified a preference for fractals with a specific amount of unpredictability within the patterns. Although the current study included some randomization within the fractal patterns to generate the trees, a combination of this preferred unpredictability in fractal patterns and fractal dimensionality of D 2.3 may result in stronger differences between nature environments. Moreover, other visual stimuli, like the predominating colors in nature (greens, blues and browns) could also play a role in the viewers preference for certain nature environments [10], as research suggests that color palettes have various emotional effects on people, like calmness or negativity [2]. From the perspective of ART, fractals could be viewed as being more strongly related to impressions of the Coherence of the scene (as indicated by results form study 1), but less strongly to the other items of the PRS [12][20]. Fractals could help the viewer to make sense of the environment, as is explained by PFA [19], but do not necessarily represent information within the environment for the user to relate to [12][20]. 
A challenge for the design of, and future research on restorative virtual nature environments, then, is to create scenes that embed fractals in a more naturalistic way. With the current scenes, framing them as a 'winter landscape' could already provide a more naturalistic contextualization. Creating more realistic virtual nature scenery, such as adding leaves, wind (i.e. motion of branches), ground vegetation, and lighting effects might result in environments that are more strongly restorative compared to the minimalistic environment presented in the present studies. The balancing act here is of attempting to isolate quantifiable factors and creating believable environments. One approach would be to take inspiration from abstracted representations of nature such as those found in paintings (e.g. Cezanne, Van Gogh), comics (e.g. Tintin), cartoons (e.g. Disney) and video games (e.g. The Legend of Zelda the Windwaker, Firewatch). Such environments could still come across as engaging and believable while at the same time offering methods of quantifying underlying factors such as fractals.

The studies presented here offer some tentative evidence for the involvement of fractals in restorative effects of (virtual) nature, but likely do so with other qualities of nature. Future research can build on these studies to further investigate parameters that enable designers to optimize virtual nature environments for the benefit of human well-being, taking into account the recommendations outlined here.

\section{ACKNOWLEDGEMENTS}

The authors gratefully acknowledge Matthijs Noordzij and Thomas van Rompay for their help and advice during the project, as well as all the participants that took part in the study.

\section{REFERENCES}

[1] Aks, D. J., \& Sprott, J. C. (1996). Quantifying Aesthetic Preference for Chaotic Patterns. Empirical Studies of the Arts, 14(1), 1-16. https://doi.org/10.2190/6V31-7M9R-T9L5-CDG9

[2] Bartram, L., Patra, A., \& Stone, M. (2017). Affective Color in Visualization. In Proceedings of the $2017 \mathrm{CHI}$ Conference on Human Factors in Computing Systems (pp. 1364-1374). New York, NY, USA: ACM. https://doi.org/10.1145/ 3025453.3026041

[3] Behrend, T. S., Sharek, D. J., Meade, A. W., \& Wiebe, E. N. (2011). The viability of crowdsourcing for survey research. Behavior Research Methods, 43(3), 800-813. https://doi.org/10.3758/s13428-011-0081-0
[4] Boucsein, W. (2012). Electrodermal Activity. Springer Science \& Business Media.

[5] Braithwaite, J. J., Watson, D. G., Jones, R., \& Rowe, M. (2013). A guide for analysing electrodermal activity (EDA) \& skin conductance responses (SCRs) for psychological experiments. Psychophysiology, 49(1), 1017-1034.

[6] Brouwer, A.-M., \& Hogervorst, M. A. (2014). A new paradigm to induce mental stress: the Singa-Song Stress Test (SSST). Frontiers in Neuroscience, 8. https://doi.org/10.3389/fnins.20 14.00224

[7] Camm, A. J., Malik, M., Bigger, J. T., Breithardt, G., Cerutti, S., Cohen, R. J., ... Singer, D. (1996). Heart rate variability. Standards of measurement, physiological interpretation, and clinical use. EUROPEAN HEART JOURNAL, 17(3), 354-381.

[8] de Kort, Y. A. W., Meijnders, A. L., Sponselee, A. A. G., \& IJsselsteijn, W. A. (2006). What's wrong with virtual trees? Restoring from stress in a mediated environment. Journal of Environmental Psychology, 26(4), 309-320. https://doi.org/10. 1016/j.jenvp.2006.09.001

[9] Delaney, J. P. A., \& Brodie, D. A. (2000). Effects of Short-Term Psychological Stress on the Time and Frequency Domains of Heart-Rate Variability. Perceptual and Motor Skills, 91(2), 515-524. https://doi.org/10.2466/pms.2000.91.2.515

[10] Depledge, M. H., Stone, R. J., \& Bird, W. J. (2011). Can Natural and Virtual Environments $\mathrm{Be}$ Used To Promote Improved Human Health and Wellbeing? Environmental Science \& Technology, 45(11), 4660-4665. $\quad$ https://doi .org/10.1021/es $103907 \mathrm{~m}$

[11] Frongillo, R. M., Lock, E., \& Brown, D. A. (2007). Symmetric fractal trees in three dimensions. Chaos, Solitons \& Fractals, 32(2), 284-295.

https://doi.org/10.1016/i.chaos.2006.04.036

[12] Gimblett, H. R., Itami, R. M., \& Fitzgibbon, J. E. (1985). Mystery in an Information Processing Model of Landscape Preference. Landscape Journal, 4(2), 87-95. https://doi.org/10.3368/ lj.4.2.87

[13] Hagerhall, C. M., Laike, T., Taylor, R. P., Küller, M., Küller, R., \& Martin, T. P. (2008). Investigations of Human EEG Response to Viewing Fractal Patterns. Perception, 37(10), 1488-1494. https://doi.org/10.1068/p5918

[14] Hagerhall, C. M., Purcell, T., \& Taylor, R. (2004). Fractal dimension of landscape silhouette outlines as a predictor of landscape preference. Journal of Environmental Psychology, 24(2), 247255.

https://doi.org/10.1016/j.jenvp.2003.12. 
[15] Hartig, T., Kaiser, F. G., \& Bowler, P. A. (1997). Further development of a measure of perceived environmental restorativeness. Institutet för bostads-och urbanforskning.

[16] Hoaglin, D. C., \& Iglewicz, B. (1987). FineTuning Some Resistant Rules for Outlier Labeling. Journal of the American Statistical Association, 82(400), 1147-1149. https://doi.org/ $\underline{10.1080 / 01621459.1987 .10478551}$

[17] Hoaglin, D. C., Iglewicz, B., \& Tukey, J. W. (1986). Performance of Some Resistant Rules for Outlier Labeling. Journal of the American Statistical Association, 81(396), 991-999. https://doi.org/10.1080/01621459.1986.10478363

[18] Jones, J. K., Farley, H., \& Murphy, A. (2018). Virtual Worlds as Restorative Environments. In Authentic Virtual World Education (pp. 45-59). Springer, Singapore. https://doi.org/10.1007/978981-10-6382-4 4

[19] Joye, Y. (2007). Architectural lessons from environmental psychology: The case of biophilic architecture. Review of General Psychology, 11(4), 305-328. https://doi.org/10.1037/1089$\underline{2680.11 .4 .305}$

[20] Kaplan, S. (1995). The restorative benefits of nature: Toward an integrative framework. Journal of Environmental Psychology, 15(3), 169-182. https://doi.org/10.1016/0272-4944(95)90001-2

[21] Kaplan, R., \& Kaplan, S. (1989). The Experience of Nature: A Psychological Perspective. CUP Archive.

[22] Korpela, K. M., Hartig, T., Kaiser, F. G., \& Fuhrer, U. (2001). Restorative Experience and Self-Regulation in Favorite Places. Environment and Behavior, 33(4), 572-589. https://doi.org/ $\underline{10.1177 / 00139160121973133}$

[23] Lechtzin, N., Busse, A. M., Smith, M. T., Grossman, S., Nesbit, S., \& Diette, G. B. (2010). A Randomized Trial of Nature Scenery and Sounds Versus Urban Scenery and Sounds to Reduce Pain in Adults Undergoing Bone Marrow Aspirate and Biopsy. The Journal of Alternative and Complementary Medicine, 16(9), 965-972. https://doi.org/10.1089/acm.2009.0531

[24] Lee, J., Park, B.-J., Tsunetsugu, Y., Kagawa, T., \& Miyazaki, Y. (2009). Restorative effects of viewing real forest landscapes, based on a comparison with urban landscapes. Scandinavian Journal of Forest Research, 24(3), 227-234. https://doi.org/10.1080/02827580902903341

[25] Mandelbrot, B. B. (1982). The fractal geometry of nature (Vol. 982). Freeman, San Francisco.

[26] Purcell, T., Peron, E., \& Berto, R. (2001). Why do Preferences Differ between Scene Types?
Environment and Behavior, 33(1), 93-106. https://doi.org/10.1177/00139160121972882

[27] Salahuddin, L., Cho, J., Jeong, M. G., \& Kim, D. (2007). Ultra Short Term Analysis of Heart Rate Variability for Monitoring Mental Stress in Mobile Settings. In 2007 29th Annual International Conference of the IEEE Engineering in Medicine and Biology Society (pp. 4656-4659). https://doi.org/10.1109//EMBS.2007.4353378

[28] Stamps, A. E. (2004). Mystery, complexity, legibility and coherence: A meta-analysis. Journal of Environmental Psychology, 24(1), 1-16. https://doi.org/10.1016/S0272-4944(03)00023-9

[29] Taylor, R., Spehar, B., Hagerhall, C., \& Van Donkelaar, P. (2011). Perceptual and Physiological Responses to Jackson Pollock's Fractals. Frontiers in Human Neuroscience, 5. https://doi.org/10.3389/fnhum.2011.00060

[30] Ulrich, R. S. (1979). Visual landscapes and psychological well-being. Landscape Research, 4(1), 17-23. https://doi.org/10.1080/014263979 $\underline{08705892}$

[31] Ulrich, R. S. (1981). Natural Versus Urban Scenes: Some Psychophysiological Effects. Environment and Behavior, 13(5), 523-556. https://doi.org/10.1177/0013916581135001

[32] Ulrich, R. S., Simons, R. F., Losito, B. D., Fiorito, E., Miles, M. A., \& Zelson, M. (1991). Stress recovery during exposure to natural and urban environments. Journal of Environmental Psychology, 11(3), 201-230. https://doi.org/ 10.1016/S0272-4944(05)80184-7

[33] Valtchanov, D., Barton, K. R., \& Ellard, C. (2010). Restorative Effects of Virtual Nature Settings. Cyberpsychology, Behavior, and Social Networking, 13(5), 503-512. https://doi.org/10. 1089/cyber.2009.0308

[34] van Rompay, T. J. L., \& Jol, T. (2016). Wild and free: Unpredictability and spaciousness as predictors of creative performance. Journal of Environmental Psychology, 48, 140-148. https: //doi.org/10.1016/j.jenvp.2016.10.001

[35] Yang, B. (1989). A cross-cultural comparison of preference for Korean, Japanese and Western landscape styles. 